\title{
Variable predictors of acute pulmonary embolism recurrence with duration of follow-up
}

\author{
Yi-Lan Yang ${ }^{1 \#}$, Ping Yuan ${ }^{1 \#}$, Chuan-Yu Wang ${ }^{1 *}$, Bigyan Pudasaini ${ }^{1}$, Yuan Li ${ }^{1}$, Yan-Zhe Yu ${ }^{2}$, Qin-Hua Zhao ${ }^{1}$, \\ Lan Wang ${ }^{1}$, Su-Gang Gong ${ }^{1}$, Rong Jiang ${ }^{1}$, Wen-Hui Wu ${ }^{1}$, Jing $\mathrm{He}^{1}$, Jian Guo ${ }^{1}$, Ci-Jun Luo ${ }^{1}$, Hong-Ling Qiu ${ }^{1}$, \\ Chang Chen ${ }^{3}$, Jin-Ling Li ${ }^{1}$, Jin-Ming Liu ${ }^{1}$
}

${ }^{1}$ Department of Cardio-Pulmonary Circulation, Shanghai Pulmonary Hospital, Tongji University, School of Medicine, Shanghai 200433, China; ${ }^{2}$ Department of Respiratory Medicine, Nanjing Drum Tower Hospital, Nanjing 210000, China; ${ }^{3}$ Department of Thoracic Surgery, Shanghai Pulmonary Hospital, Tongji University, School of Medicine, Shanghai 200433, China

Contributions: (I) Conception and design: YL Yang, JL Li, JM Liu; (II) Administrative support: JM Liu, YL Yang, P Yuan; (III) Provision of study materials or patients: CY Wang, SG Gong, J Guo, JM Liu; (IV) Collection and assembly of data: YL Yang, P Yuan, JM Liu; (V) Data analysis and interpretation: All authors; (VI) Manuscript writing: All authors; (VII) Final approval of manuscript: All authors.

\#These authors contributed equally to this work.

Correspondence to: Prof. Jin-Ming Liu, MD; Jin-Ling Li, MD. Shanghai Pulmonary Hospital Affiliated to Tongji University, No. 507 Zhengmin Road, Shanghai 200433, China. Email: jinmingliu@tongji.edu.cn; chnfl93@hotmail.com.

Background: Acute pulmonary embolism (PE) is a critical disease and often leads to a high mortality and morbidity. Several studies have identified predictors of PE recurrence, but whether these predictors have prognostic value and how they vary during varied follow-up periods remain unclear.

Methods: We retrospectively assessed the occurrence of recurrent PE and the survival time of patients with a diagnosis of acute PE at Shanghai Pulmonary Hospital from May 2007 to May 2018. Potential predictors of recurrent PE were evaluated at different points (1, 3, 6, 12, 24, 60 and 120-month) during a long-term follow-up for each patient. Patients were stratified into two groups by gender to analyze the impact of sex in period-guided prognostic prediction. Receiver operating characteristic curve analysis, survival analysis and multivariate Cox proportional hazards analysis were implemented as statistical analysis methods.

Results: In total, 597 acute PE patients were included, of whom 62 reported a PE recurrence. Male patients tend to have a lower risk of PE recurrence than female patients during 3- to 60-month follow-up period but have a higher risk of $\mathrm{PE}$ recurrence than female patients during 120-month follow-up period. The independent predictors of recurrence-free survival varied among different follow-up periods: In all patients, diabetes was an independent predictor only within 30 days follow-up period and female was considered as an independent predictor during 3- to 120-month follow-up period. Among male patients, hyperlipidemia and Log D-dimer (cut-off value =3.436) was observed as a predictor of recurrent PE within 6-month and over 12-month follow-up respectively. However, there is no unified independent prognostic indicator for female patients identified.

Conclusions: In the early stage of follow-up, male PE patients have better prognosis, but with the extension of follow-up, female PE patients have better prognosis. The independent predictors of recurrencefree survival vary in different follow-up periods in PE patients when stratified based on gender and associated medical conditions.

Keywords: Pulmonary embolism (PE); prognostic value; recurrent predictors

Submitted Jul 02, 2019. Accepted for publication Jan 06, 2020.

doi: $10.21037 /$ jtd.2020.01.27

View this article at: http://dx.doi.org/10.21037/jtd.2020.01.27 


\section{Introduction}

Acute pulmonary embolism (PE) due to endogenous or exogenous thrombosis in the pulmonary arterial trunk or its branch is a cardiovascular emergency (1-5) causing substantial morbidity and mortality (6). Acute PE is associated with a certain rate of recurrence and prolonged anticoagulant therapy subsequently. Given that longterm anticoagulant treatment increases the risk of major bleeding, it greatly enlarges the burden of social as well as family medical care cost (7). Therefore, it is crucial to prevent PE recurrence by predicting it through different achievable indicators along the follow-up period after an acute PE happens.

The prognosis is considered favorable in most of the patients after the initial onset of $\mathrm{PE}$, but the recurrence of $\mathrm{PE}$ causes a severe clinical complication. Moreover, there is a paucity of prognostic data regarding $\mathrm{PE}$ recurrence in Chinese population most predominantly. The challenge perhaps involves balancing of the risks of recurrence of $\mathrm{PE} /$ venous thromboembolism (VTE) against the risks of longterm therapy especially in older patients. Several studies have explored several novel predictive parameters of $\mathrm{PE}$ recurrence. High creatinine kinase isoenzyme MB (CK-MB) was found to be associated with a high risk of in-hospital mortality during a 30-month follow-up period (8). Another study indicated diabetes as an independent predictor of deep vein thrombosis (DVT) recurrence during a median follow-up period of 33 months (9). Bjøri and his colleagues found that a low level of D-dimer $(\leq 1,500 \mathrm{ng} / \mathrm{mL})$ during the first VTE diagnosis was associated with low recurrence risk during a median follow-up period of 47 months (10). Different predictors have been suggested at different follow up period in different studies which displays a knowledge gap in this field.

It remains unclear as to the association between followup period length and the different $\mathrm{PE}$ recurrence predictors. Thus, the present study aimed to explore whether these predictors maintain their prognostic value in varied followup periods.

\section{Methods}

\section{Study variables}

This study included 597 patients (319 males and 278 females) over 18 years old who were diagnosed with acute PE in the Shanghai Pulmonary Hospital from May 2007 to May 2018. The registration information included clinical variables, biochemical indexes, risk factors for VTE, comorbid diseases, clinical symptoms and signs, risk stratification and treatment of all patients were evaluated at baseline. Recent surgery was defined as those patients who had undergone an operation in the 2 months prior to $\mathrm{PE}$ diagnosis. The risk stratification was assessed by the algorithm of the 2019 European Society of Cardiology (ESC) guidelines of PE (7). Besides, a logarithmic transformation of D-dimer (Log D-dimer) was performed.

The diagnosis of PE in this study referred to the 2019 ESC guidelines which recommends the PE diagnosis based on the sequential use of clinical or pre-test probability assessment, D-dimer measurement, computed tomography pulmonary angiography (CTPA), ventilation perfusion scan, or leg ultrasonography $(7,11,12)$. The patients with insufficient data, APE of non-thrombus origin (fat or tumor emboli) and patients with active cancer (newly diagnosed cancer or metastatic cancer) or thrombophilia were excluded.

\section{Outcomes}

The primary outcome and secondary endpoint of this study was the incidence of recurrent PE and recurrent DVT respectively. Two investigators (authors Yi-Lan Yang and Ping Yuan) independently adjudicated the cause of PE or DVT recurrence (13). These investigators defined recurrent $\mathrm{PE}$ as the presence of a new perfusion defect which involved $75 \%$ or more of a lung segment on V/Q scintigraphy, or a new intraluminal filling defect, or an extension of a filling defect on CTPA $(14,15)$. Recurrent DVT was defined as the appearance of a new non-vein segment, or a 4-mm or more increase in the previous diameter of a thrombosis that is compressible on complete compression ultrasound $(15,16)$.

\section{Treatment and follow-up}

All patients have received standardized anticoagulant treatment for 3-6 months after acute PE. There is no universal consensus on the treatment duration of anticoagulation for recurrent VTE prevention $(17,18)$. Thus, whether to prolong the treatment was determined by professional clinicians based on etiology or disease status. After discharge, all data of patients were obtained during their visits at outpatient clinics or by telephone interviews. Any signs or symptoms suggesting PE or DVT recurrences were noted. Patients with suspected recurrent VTE were confirmed via diagnostic testing immediately. And potential 
predictors of recurrent $\mathrm{PE}$ were evaluated at different points $(1,3,6,12,24,60,84,86,96$ and 120-month) during a long-term follow-up for each patient.

\section{Statistical analysis}

Continuous variables were expressed as mean with standard deviation (SD) or medians (and interquartile rang), and categorical variables were expressed as absolute number. Comparisons were performed using t-tests or MannWhitney U-test for continuous variables and chi-square or Fisher's exact tests for categorical variables. The impact of sex (female vs. male) on prognosis was evaluated using univariate and multivariable cox proportional hazards analyses. Age, BMI and/or sex (model 1 and model 2) were forced in models to adjust the multivariate analysis. Receiver operating characteristic (ROC) curves for independent parameters were drawn and the areas under the curves were calculated. For a specific parameter, the cutoff level that resulted in the highest product of sensitivity and specificity was considered to be an optimal cutoff value for prognostication. Kaplan-Meier methods were used to estimate the outcome of time to $\mathrm{PE}$ recurrence, and disparities between the sexes were assessed with long-rank test. Statistical significance was defined as the two-tailed $\mathrm{P}$ value of $<0.05$ for all analyses. Statistical analysis was performed using SPSS (Statistic Package for Social Science, Chicago, IL) version 20.0 and GraphPad Prism (San Diego, CA, USA) version 6.0.

\section{Results}

\section{Characteristics of study variables}

A total of 597 acute PE patients who met the inclusion criteria were included, and 319 (53.4\%) of them were men. The mean duration of follow-up was $24 \pm 18$ months, with PE reoccurred in 24 men and 38 women during the whole follow up period. Only 5 patients were DVT recurrence. The baseline information was presented in Table 1.

In males, compared with those without a PE recurrence, patients with a $\mathrm{PE}$ recurrence were estimated at a higher Log D-dimer level, with a larger proportion of altered mental status (disorientation, stupor or coma) and syncope. In contrast, female patients with $\mathrm{PE}$ recurrence were associated with higher prevalence of DVT than those without PE recurrence. Among all patients with PE recurrence, male patients had significantly higher serum creatinine levels and higher prevalence of altered mental status than female patients (Table 1). In non-recurrent PE group, males had significantly higher serum creatinine levels, higher prevalence of DVT, acute infection, diabetes, other lung diseases, cough and sputum, and hemoptysis compared to females.

\section{Survival analysis in different follow-up periods}

Sixty-two (10.4\%) patients reported a PE recurrence. The recurrence rate was $13.7 \%(38 / 278)$ in female group and $7.5 \%$ (24/319) in male group. There is an intersection of the recurrence-free survival curve between male and female $\mathrm{PE}$ patients. In the first 30-days follow-up period after acute $\mathrm{PE}$, there was no significant difference with regard to recurrence-free survival rate $(\mathrm{P}=0.484)$ between men and women (Figure S1). After that, the recurrence-free survival rate in female patients dropped to a lower number than that of male patients and this difference remained at 3-, 6-, 12-, 24-, 60- and 84-month follow-up. During 86-month follow-up, male patients had the same prognosis as female patients $(\mathrm{P}=0.053)$ (Figure $S 1 E)$. In contrast, female patients showed significantly better recurrencefree survival rates than the male patients during 96 to 120-month follow-up (Figure S1F and Figure 1).

\section{Predictive factors of PE recurrence during different follow- up periods}

The multivariate cox proportional hazards analysis revealed that (I) for the whole cohort, female sex and higher Log D-dimer were independent risk factors of poorer recurrence-free survival; (II) for male patients, age and Log D-dimer were considered as independent risk factors of recurrence-free survival; (III) but no significant risk factors of recurrence-free survival had been identified among female patients (Table 2).

To analyze the predictors of sex disparities during the follow-up period, the prevalence of diabetes was regarded as an independent predictor of 30-days $\mathrm{PE}$ recurrence probability in all patients (Table S1). As shown in Table 3 and Table S1, female sex was estimated as an independent predictor of $\mathrm{PE}$ recurrence probability during all followup periods (3-, 6-, 12-, 24-, 60- and 120-month) in all patients. Hyperlipidemia and Log D-dimer was identified as an independent predictor of $\mathrm{PE}$ recurrence in male patients during the 6-month follow-up and during the medium and long-term (12-, 24-, 60-, 120-month), 
Table 1 Baseline characteristics of patients with acute symptomatic pulmonary embolism

\begin{tabular}{|c|c|c|c|c|c|}
\hline Variables & $\begin{array}{l}\text { All patients } \\
\quad(n=597)\end{array}$ & \multicolumn{2}{|c|}{ Male } & \multicolumn{2}{|c|}{ Female } \\
\hline Age, years & $61 \pm 15$ & $68 \pm 13$ & $60 \pm 16$ & $61 \pm 12$ & $62 \pm 13$ \\
\hline $\mathrm{BMI}, \mathrm{kg} / \mathrm{m}^{2}$ & $24(20-28)$ & $24(22-27)$ & $26(23-33)$ & $22(17-25)$ & $22(18-25)$ \\
\hline \multicolumn{6}{|l|}{ Biochemical indexes } \\
\hline Log D-dimer & $3.0(2.6-3.4)$ & $3.3(2.9-3.6)$ & $2.9(2.6-3.3)^{\mathrm{a}}$ & $2.8(2.4-3.5)$ & $3.0(2.6-3.4)$ \\
\hline Creatinine, $\mu \mathrm{mol} / \mathrm{L}$ & $65(54-77)$ & $75(67-83)$ & $72(63-82)$ & $52(44-60)^{b}$ & $55(49-65)^{c}$ \\
\hline NT-proBNP, ng/L & $129(47-551)$ & $216(48-774)$ & $132(44-654)$ & $90(47-489)$ & $111(54-462)$ \\
\hline \multicolumn{6}{|l|}{ Risk factors for VTE } \\
\hline History of DVT, n [\%] & $103[17]$ & 7 [29] & 59 [20] & 9 [24] & $28[12]^{a \& c}$ \\
\hline \multicolumn{6}{|l|}{ Comorbid diseases } \\
\hline Acute infection, $\mathrm{n}[\%]$ & $307[51]$ & $12[50]$ & $172[58]$ & $11[29]$ & $112[47]^{\mathrm{c}}$ \\
\hline Altered mental status $\dagger, \mathrm{n}[\%]$ & $28[5]$ & $4[17]$ & ${ }^{11}[4]^{\mathrm{a}}$ & ${ }^{1}[3]^{\mathrm{b}}$ & $12[5]$ \\
\hline Atrial Fibrillation, n [\%] & $25[4]$ & $0[0]$ & $16[5]$ & $3[8]$ & 6 [3] \\
\hline Hypertension, $\mathrm{n}$ [\%] & $195[33]$ & 7 [29] & $88[30]$ & $13[34]$ & $87[36]$ \\
\hline Diabetes, n [\%] & $57[10]$ & $2[8]$ & $36[12]$ & $2[5]$ & $17[7]^{\mathrm{c}}$ \\
\hline Cough and sputum, n [\%] & $179[30]$ & $10[42]$ & $102[35]$ & 12 [32] & $55[23]^{c}$ \\
\hline Chest pain, n [\%] & $91[15]$ & $3[13]$ & $51[17]$ & $6[16]$ & $31[13]$ \\
\hline Dyspnea, n [\%] & 248 [42] & 9 [38] & 124 [42] & $18[47]$ & $97[40]$ \\
\hline Hemoptysis, n [\%] & $53[9]$ & $4[17]$ & 37 [13] & $3[8]$ & $9[4]^{c}$ \\
\hline Syncope, n [\%] & $28[5]$ & $3[13]$ & $10[3]^{\mathrm{a}}$ & $2[5]$ & $13[5]$ \\
\hline sPESI $\geq 1$ point(s), $n$ [\%] & $296[50]$ & $13[54]$ & $146[49]$ & 15 [39] & $122[51]$ \\
\hline \multicolumn{6}{|l|}{2019 ESC guidelines algorithm } \\
\hline High-risk, n [\%] & $59[10]$ & $3[13]$ & $28[9]$ & $6[16]$ & $22[9]$ \\
\hline Intermediate high-risk, n [\%] & $155[26]$ & 7 [29] & $83[28]$ & 9 [24] & $56[23]$ \\
\hline Intermediate low-risk, n [\%] & 231 [39] & 8 [33] & $108[37]$ & $12[32]$ & $103[43]$ \\
\hline Low-risk, n [\%] & $152[25]$ & $6[25]$ & $76[26]$ & $11[29]$ & 59 [25] \\
\hline
\end{tabular}

Table 1 (continued) 
Table 1 (continued)

\begin{tabular}{|c|c|c|c|c|c|}
\hline Variables & $\begin{array}{l}\text { All patients } \\
\quad(\mathrm{n}=597)\end{array}$ & \multicolumn{2}{|c|}{ Male } & \multicolumn{2}{|c|}{ Female } \\
\hline \multicolumn{6}{|l|}{ Treatment } \\
\hline Vitamin-K antagonist, $\mathrm{n}$ [\%] & 368 [62] & $16[67]$ & $169[57]$ & $31[82]$ & $152[63]$ \\
\hline
\end{tabular}

Values are mean (SD) or median (interquartile range). $\dagger$, altered mental status, disorientation, stupor or coma; $\ddagger$, lung disease, pulmonary infarction, respiratory failure, Chronic lung disease; ${ }^{a} \mathrm{P}<0.05$, nonrecurrence vs. recurrence. ${ }^{b} \mathrm{P}<0.05$, recurrence female patients vs. recurrence male patients. ${ }^{c} \mathrm{P}<0.05$, nonrecurrence female patients vs. nonrecurrence male patients. BMI, Body mass index; CK-MB, creatinine kinase isoenzyme MB; DVT, deep vein thrombosis; Log D-dimer, a logarithmic transformation of the D-dimer; NT-pro BNP, nitrogen terminal brain natriuretic peptide; NOAC, non-vitamin K-dependent oral anticoagulant; Recent Surgery, nearly two months of surgery or brake history; sPESI, simplified Pulmonary Embolism Severity Index.

respectively. In female patients, a history of DVT or acute infection was proved to be an independent predictor of short to medium-term (3-, 6-, 12-month) PE recurrence probability (Table 3 and Table S1). However, there was no independent predictors at both medium- and long-term (24-, 60-, 120-month) PE recurrence probability in female patients (Table 3).

\section{Receiver operating characteristic curve analysis}

ROC curve was implemented to analyze the cut-off value of $\log \mathrm{D}$-dimer as male's independent predictor of PE recurrence probability in different follow-up periods (Figure 2 and Table 4). Male patients were divided into two subgroups according to this cut-off value in Figure 3. In general, the cut-off value of $\log \mathrm{D}$-dimer in male patients was $3.436 \mathrm{ng} / \mathrm{mL}$. Patients with a value $<3.436 \mathrm{ng} / \mathrm{mL}$ showed a significantly higher cumulative PE recurrencefree survival rate and a lower PE recurrence probability than those with $\geq 3.436 \mathrm{ng} / \mathrm{mL}$ during $12-, 24-, 60$ - and 120-month follow-up (Figure 3).

\section{Discussion}

In the present study, we observed male PE patients have a better prognosis during the early stage of follow-up period, but with the extension of follow-up period, female PE patients have better prognosis. So there is an intersection of the eventfree survival curve between male and female PE patients. The independent predictors of recurrence-free survival vary in different follow-up periods in PE patients when stratified based on gender and associated medical conditions.

According to the 2019 ESC guidelines of PE, there is an extensive collection of predisposing environmental and genetic factors for VTE (7). These includes weak to strong predisposing factors for VTE $(7,19-21)$. While, in the present study, patients with active cancer and thrombophilia were excluded, and some risk factors are also excluded due to the limited sample size. So we do not focus on the all risk factors reported in the 2019 ESC guidelines. Table 1 presented some risk factors, and univariate analysis found that patients with a previous history of DVT and infection were considered as risk factors for PE recurrence. These indicators were consistent with the 2019 ESC guidelines of PE (7). Interestingly, some of the PE risk factors found in the study were not mentioned in the guidelines.

Diabetes as a risk factor of DVT and PE development has been well documented by multiple studies. Chung et al. proved that patients with diabetes carried greater risks of developing VTE than general population (22). Moreover, diabetes was discovered as an independent predictor for recurrent DVT in Piazza et al.'s study (9). However, study on the association between diabetes and increases PE recurrence is scarce. In our study, diabetes was able to predict a $\mathrm{PE}$ recurrence during a 30-day follow-up.

Despite several reports on sex differences in predicting the prognosis of PE (21,23-25), our study found that male patients had a more severe clinical status at the time of diagnosis even though they had a lower-risk and better PE recurrence-free survival rate than female patients. Male patients were more likely to develop DVT, acute infection, diabetes and lung diseases than female patients, and were 


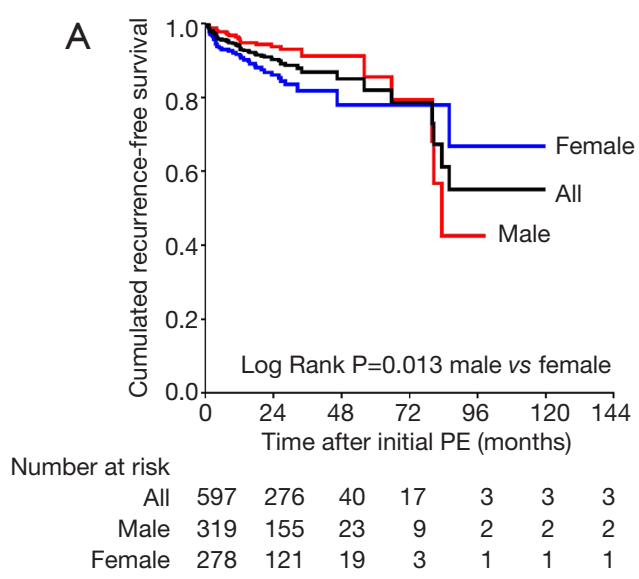

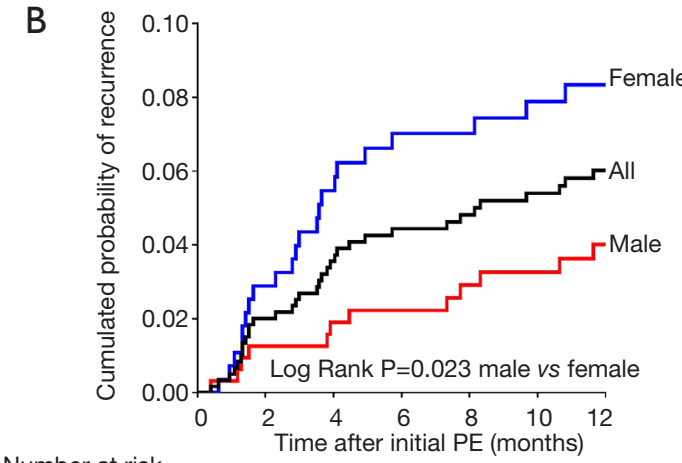

Number at risk

$\begin{array}{llllllll}\text { All } & 597 & 555 & 520 & 520 & 501 & 473 & 438\end{array}$

Male $\begin{array}{lllllll}319 & 314 & 304 & 290 & 280 & 268 & 246\end{array}$

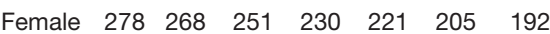

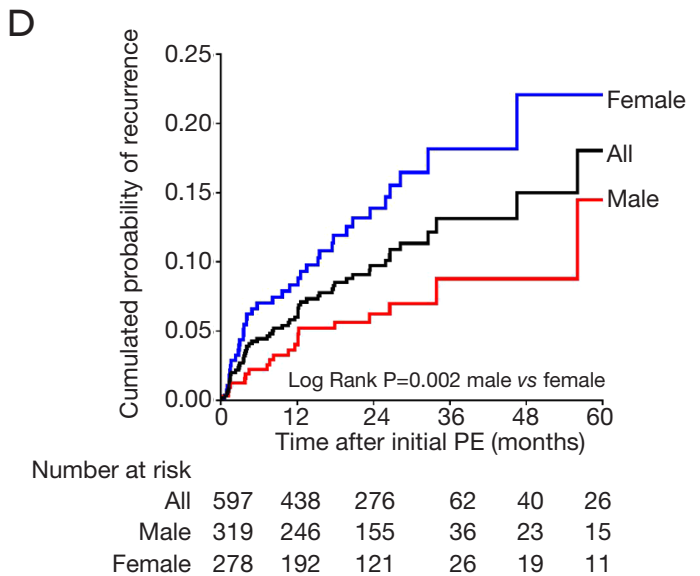

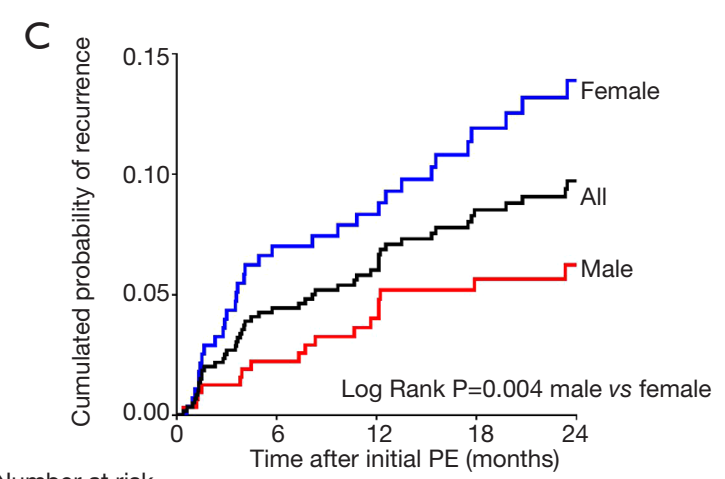

Number at risk

$\begin{array}{rrrrrc}\text { All } & 597 & 520 & 438 & 367 & 276 \\ \text { Male } & 319 & 290 & 246 & 210 & 155 \\ \text { Female } & 278 & 230 & 192 & 155 & 121\end{array}$

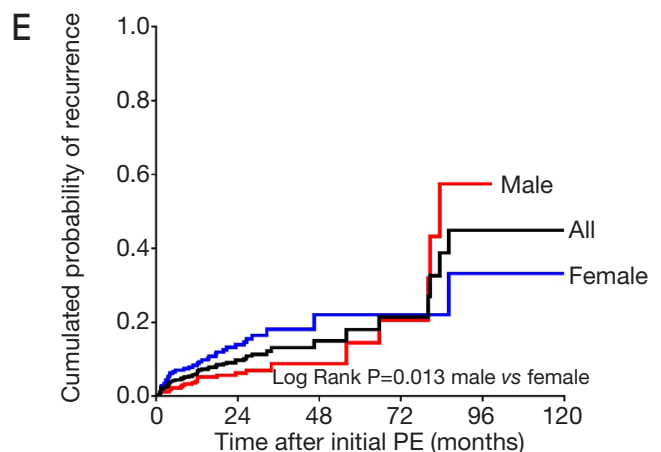

Number at risk

$\begin{array}{rrrrrrr}\text { All } & 597 & 276 & 40 & 17 & 3 & 3 \\ \text { Male } & 319 & 155 & 23 & 9 & 2 & 2 \\ \text { Female } & 278 & 121 & 19 & 8 & 1 & 1\end{array}$

Figure 1 Sex disparities in cumulative recurrence-free survival curves and recurrence probability of patients with PE. (A) The total cumulative recurrence-free survival curves; (B-E) cumulative recurrence probability during 12, 24, 60 and 120-month follow-up periods.

more likely to present a severe symptom at the time of diagnosis. Independent prognostic factors in all patients as a single group were not similar to that of independent predictors in the two sexes. And the predictors evaluated showed significant differences between the two sexes, which was not in line with that of the previous studies $(21,23,24)$.

Several clinical variables have been revealed that might determine the outcome of PE patients in the previous 
Table 2 Parameters predictive of long-term recurrence-free survival rate in enter univariate and multivariate cox proportional hazards analysis

\begin{tabular}{|c|c|c|c|c|c|}
\hline Populations studied & Variables & \multicolumn{2}{|l|}{ Univariate } & \multicolumn{2}{|c|}{ Multivariate } \\
\hline \multirow[t]{2}{*}{ All } & Female sex & $1.897(1.136-3.167)$ & 0.014 & $1.968(1.171-3.310)$ & 0.011 \\
\hline & Log D-dimer & $1.627(1.026-2.580)$ & 0.039 & $1.612(1.011-2.568)$ & 0.045 \\
\hline \multirow[t]{2}{*}{ Male } & Age & $1.039(1.003-1.076)$ & 0.034 & $3.992(1.374-11.598)$ & 0.011 \\
\hline & Altered mental status $\dagger$ & $3.653(1.055-12.647)$ & 0.041 & - & - \\
\hline Female & - & - & - & - & - \\
\hline
\end{tabular}

Values are median (interquartile range). †Altered mental status, disorientation, stupor or coma. Cl, confidence interval; Log D-dimer, a logarithmic transformation of the D-dimer.

Table 3 Parameters predictive of recurrence probability in enter univariate and multivariate Cox proportional hazards analysis

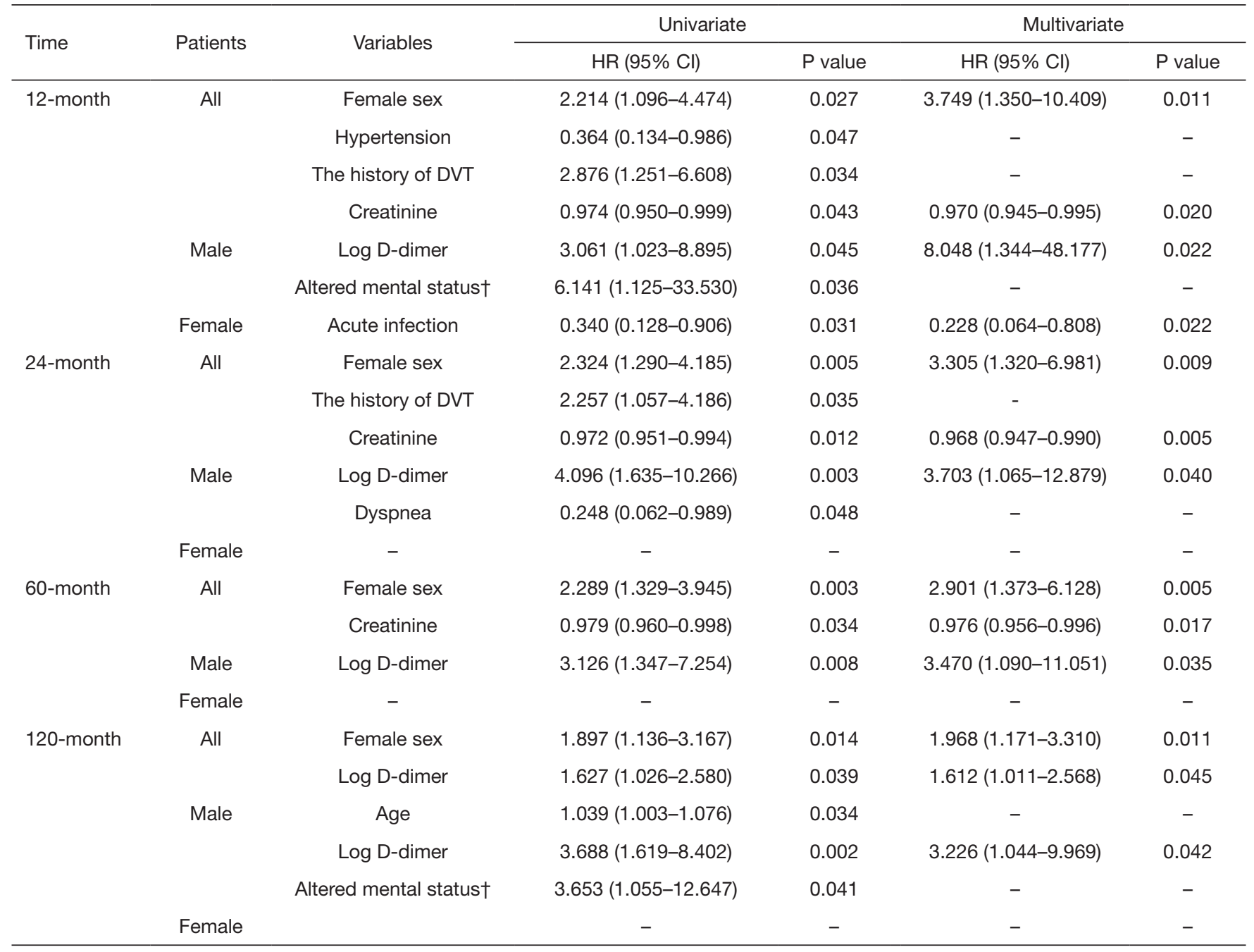

Values are median (interquartile range). †Altered mental status, disorientation, stupor or coma. Cl, confidence interval; DVT, deep vein thrombosis; Log D-dimer, a logarithmic transformation of D-dimer. 
A

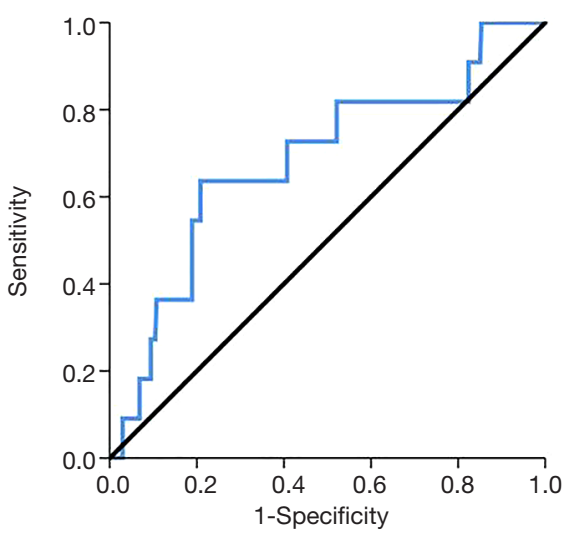

C

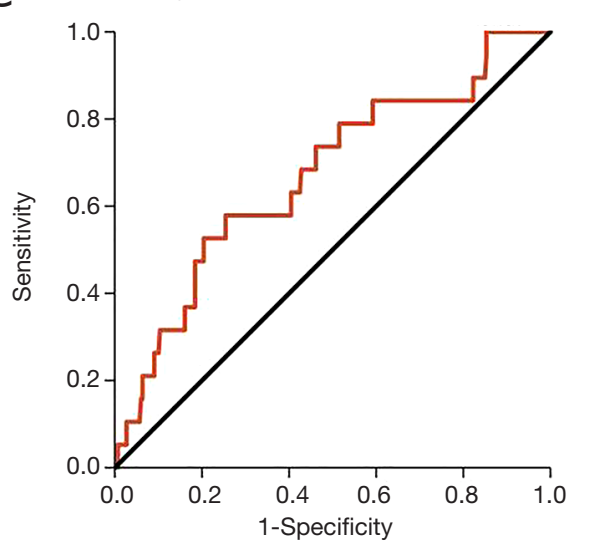

B

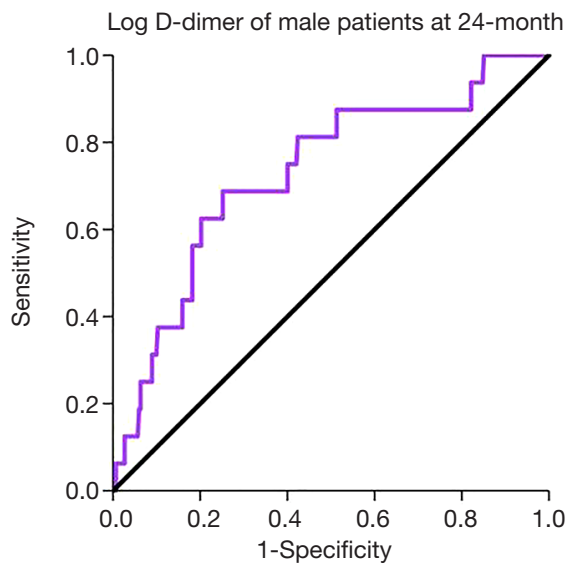

D

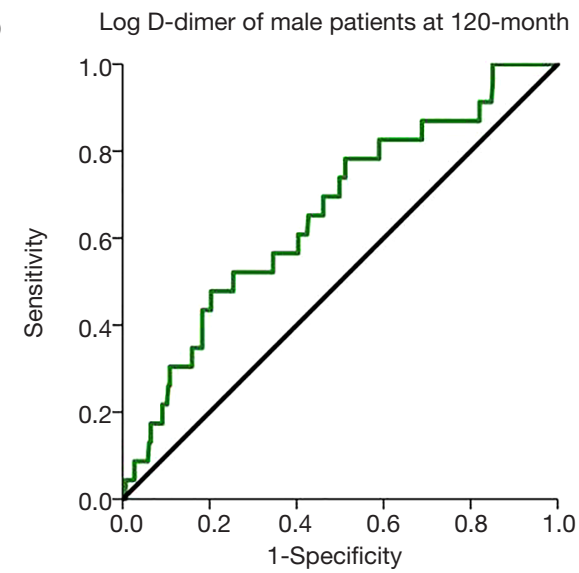

Figure 2 Receiver operating characteristic (ROC) curves of Log D-dimer in male patients. (A-D) Roc curve of Log D-dimer in male patients during 12, 24, 60 and 120-month follow-up periods.

Table 4 Receiver-operating characteristics of $\log \mathrm{D}$-dimer in male patients

\begin{tabular}{|c|c|c|c|c|c|c|}
\hline Time & Area & Cut-off value & Sensitivity & Specificity & $95 \% \mathrm{Cl}$ & $P$ value \\
\hline 12-months & 0.683 & 3.436 & 0.636 & 0.788 & $0.513-0.852$ & 0.039 \\
\hline 24-months & 0.730 & 3.436 & 0.625 & 0.798 & $0.601-0.858$ & 0.002 \\
\hline 60-months & 0.670 & 3.436 & 0.526 & 0.796 & $0.542-0.799$ & 0.013 \\
\hline 120-months & 0.657 & 3.436 & 0.478 & 0.797 & $0.543-0.771$ & 0.012 \\
\hline
\end{tabular}

studies. Our study indicated, patients with a higher Log D-dimer level, a larger proportion of altered mental status and syncope were more likely to have PE recurrence. Log D-dimer was regarded as an independent predictor of recurrence-free survival overall and particularly in male patients with $\mathrm{PE}$. The reason that Log D-dimer was not justified as a predictor in females was potentially because that estrogen uses which was reported to be associated with D-dimer level in a recent study (26). Sanchez et al. proved that altered mental state was associated with 30-days of adverse events (27), while, we only found male patients with $\mathrm{PE}$ recurrence had higher proportion of altered mental status than those with no recurrence. Although acute right heart failure was not presented in this study, the previous study indicated that increased the right heart overload due to $\mathrm{PE}$ could lead to arrhythmia and vasovagal reflex, resulting in syncope. Furthermore, the presence of syncope in PE patients showed association with severe adverse events (28-31). 
A

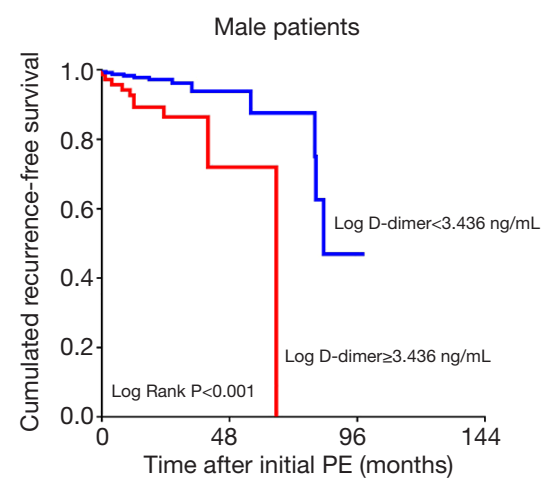

Number at risk

$\begin{array}{lllll}\text { Log D-dimer }<3.436 \mathrm{ng} / \mathrm{mL} & 249 & 240 & 239 & 236\end{array}$

$\begin{array}{lllll}\text { Log D-dimer } \geq 3.436 \mathrm{ng} / \mathrm{mL} & 70 & 60 & 59 & 59\end{array}$

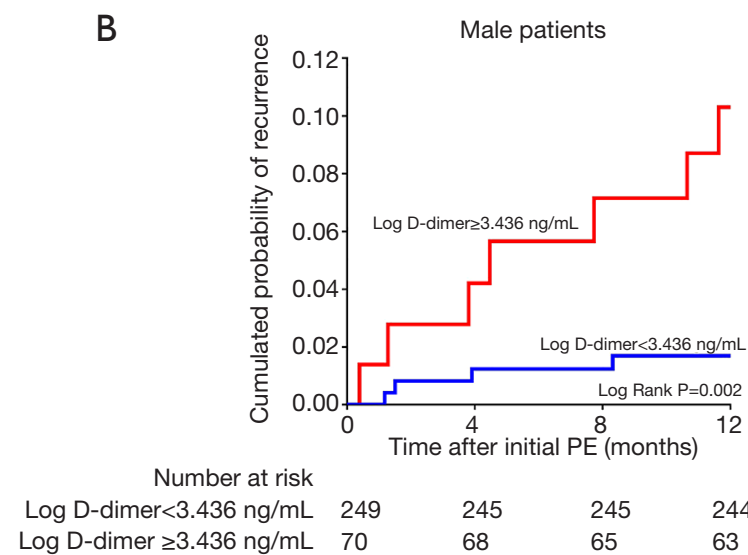

D

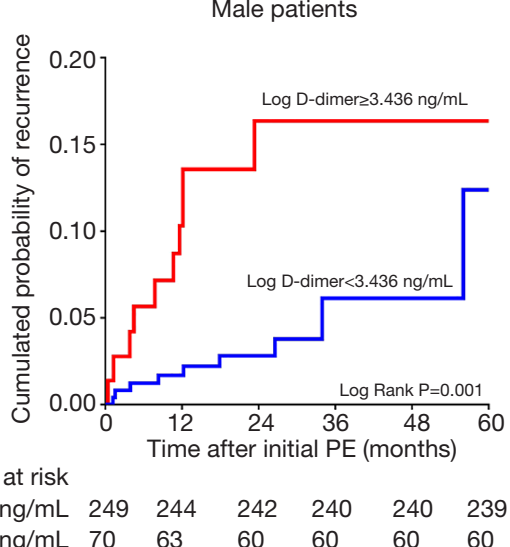

C

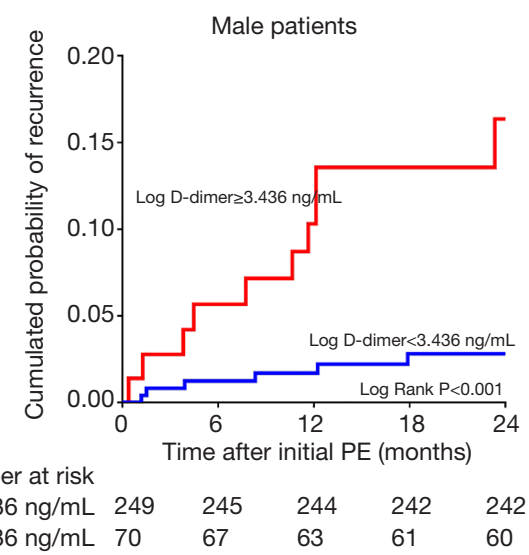

E

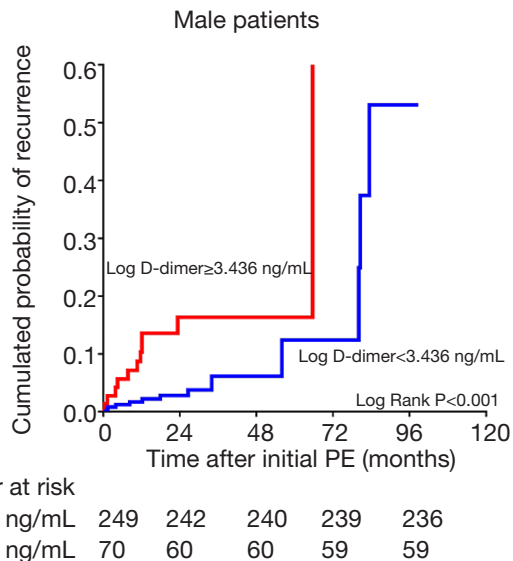

Figure 3 Kaplan-Meier cumulative recurrence-free survival curves and recurrence probability in male patients with different Log D-dimer levels. (A) The total cumulative recurrence-free survival curves between male patients with Log D-dimer value of $<3.436 \mathrm{ng} / \mathrm{mL}$ and male patients with $\log \mathrm{D}$-dimer value of $\geq 3.436 \mathrm{ng} / \mathrm{mL}$; (B-E) cumulative recurrence probability between male patients with Log D-dimer value of $<3.436 \mathrm{ng} / \mathrm{mL}$ and male patients with $\log$ D-dimer value of $\geq 3.436 \mathrm{ng} / \mathrm{ml}$ during 12, 24,60 and 120-month follow-up periods. 
Controversy exists regarding the association between sex and risk of $\mathrm{PE}$ recurrence. Our results suggest that women have a higher risk of $\mathrm{PE}$ recurrence, which is inconsistent with that of the previous studies (26). However, the underlying mechanisms should be further elucidated. The different risk of $\mathrm{PE}$ recurrence between the two sexes might be related to differences in thrombotic and fibrinolytic activities $(26,32)$. Moreover, with the extension of the follow-up periods, the prognosis of male patients tended to be worse than female patients. A larger sample size is needed to explain the changed of the recurrence-free survival rates.

Several limitations could be seen in this study. A small sample size is a major limitation and therefore the stability analyses of prognostic model were debatable and incomplete. Also, the data does not fully reflect the association between sex and diseases severity since we did not analyze the all-cause mortality and PE-related deaths. Secondly, our study did not include myocardial injury markers, such as troponin, $\beta$-natriuretic peptide (BNP) and other right ventricular parameters. Moreover, some bias may be under-evaluated including different intervention patterns (surgery, medication and others).

\section{Conclusions}

In conclusion, our findings suggest that male patients tend to have a better prognosis than female patients during 3to 60-month follow-up period, but have a worse prognosis than female patients during 120-month follow-up period. However, there is no unified independent prognostic indicator for female patients identified. Only the history of DVT and acute infection were considered as recurrence predictors in female patients within 3- and 12-month follow-up periods. Different predictors for of PE recurrence are found during different follow-up periods. These data may provide new evidences that different predictors for PE recurrence should be selected during the different follow-up periods. We believe that a standard predicting method assists in providing precious treatment, enhancing clinical judgement, reducing missed diagnosis/mis-diagnosis rates, thereby improving prognosis.

\section{Acknowledgments}

Funding: This work was supported by the Program of National Natural Science Foundation of China (81870042, 81700045 and 81600032), National Science and Technology Information System of the People's Republic of China (2018YFC1313603).

\section{Footnote}

Conflicts of Interest: The authors have no conflicts of interest to declare.

Ethical Statement: The authors are accountable for all aspects of the work in ensuring that questions related to the accuracy or integrity of any part of the work are appropriately investigated and resolved. The protocol of this study was reviewed and approved by the Ethics Committee of Shanghai Pulmonary Hospital (approval no: K18-198Y). Written informed consent has obtained from each patient for inclusion into the study and prior to the performance of any study-related procedures.

Open Access Statement: This is an Open Access article distributed in accordance with the Creative Commons Attribution-NonCommercial-NoDerivs 4.0 International License (CC BY-NC-ND 4.0), which permits the noncommercial replication and distribution of the article with the strict proviso that no changes or edits are made and the original work is properly cited (including links to both the formal publication through the relevant DOI and the license). See: https://creativecommons.org/licenses/by-nc-nd/4.0/.

\section{References}

1. Clagett GP, Anderson FA Jr, Heit J, et al. Prevention of venous thromboembolism. Chest 1995;108:312S-34S.

2. Chen TX, Pudasaini B, Guo J, et al. Sex-specific cardiopulmonary exercise testing indices to estimate the severity of inoperable chronic thromboembolic pulmonary hypertension. Int $\mathrm{J}$ Chron Obstruct Pulmon Dis 2018;13:385-97.

3. Keller K, Beule J, Balzer JO, et al. D-Dimer and thrombus burden in acute pulmonary embolism. Am J Emerg Med 2018;36:1613-8.

4. Ben Halima N, Sghaier A, Thabet H, et al. Thrombolysis for pulmonary embolism during pregnancy. About four cases. Tunis Med 2018;96:80-3.

5. Torbicki A, Perrier A, Konstantinides S, et al. Guidelines on the diagnosis and management of acute pulmonary embolism: the Task Force for the Diagnosis and Management of Acute Pulmonary Embolism of the European Society of Cardiology (ESC). Eur Heart J 2008;29:2276-315.

6. Cohen AT, Agnelli G, Anderson FA, et al. Venous thromboembolism (VTE) in Europe. The number of VTE events and associated morbidity and mortality. Thromb 
Haemost 2007;98:756-64.

7. Konstantinides SV, Meyer G, Becattini C, et al. 2019 ESC Guidelines for the diagnosis and management of acute pulmonary embolism developed in collaboration with the European Respiratory Society (ERS). Eur Heart J 2020;41:543-603.

8. Bozbay M, Uyarel H, Avsar S, et al. Creatinine kinase isoenzyme-MB: A simple prognostic biomarker in patients with pulmonary embolism treated with thrombolytic therapy. J Crit Care 2015;30:1179-83.

9. Piazza G, Goldhaber SZ, Kroll A, et al. Venous thromboembolism in patients with diabetes mellitus. Am J Med 2012;125:709-16.

10. Bjøri E, Johnsen HS, Hansen JB, et al. D-dimer at venous thrombosis diagnosis is associated with risk of recurrence. J Thromb Haemost 2017;15:917-24.

11. Righini M, Robert-Ebadi H, Le Gal G. Diagnosis of acute pulmonary embolism. J Thromb Haemost 2017;15:1251-61.

12. Huisman MV, Barco S, Cannegieter SC, et al. Pulmonary embolism. Nat Rev Dis Primers 2018;4:18028.

13. Aujesky D, Obrosky DS, Stone RA, et al. Derivation and validation of a prognostic model for pulmonary embolism. Am J Respir Crit Care Med 2005;172:1041-6.

14. Riera-Mestre A, Jiménez D, Muriel A, et al. RIETE investigators. Thrombolytic therapy and outcome of patients with an acute symptomatic pulmonary embolism. J Thromb Haemost 2012;10:751-9.

15. Remy-Jardin M, Remy J, Wattinne L, et al. Central pulmonary thromboembolism: diagnosis with spiral volumetric CT with the single-breath-hold-techniquecomparison with pulmonary angiography. Radiology 1992;185:381-7.

16. Prandoni $\mathrm{P}$, Cogo A, Bernardi E, et al. A simple approach for detection of recurrent proximal vein thrombosis. Circulation 1993;88:1730-5.

17. Kearon C. Indefinite anticoagulation after a first episode of unprovoked venous thromboembolism: yes. J Thromb Haemost 2007;5:2330-5.

18. Kearon C, Akl EA, Ornelas J, et al. Antithrombotic Therapy for VTE Disease: CHEST Guideline and Expert Panel Report. Chest 2016;149:315-52.

19. Prandoni P, Falanga A, Piccioli A. Cancer and venous thromboembolism. Lancet Oncol 2005;6:401-10.

20. Chung WS, Lin CL, Hsu WH, et al. Idiopathic venous thromboembolism: a potential surrogate for occult cancer. QJM 2014;107:529-36.

21. Tanabe Y, Yamamoto T, Murata T, et al. Gender
Differences Among Patients With Acute Pulmonary Embolism. Am J Cardiol 2018;122:1079-84.

22. Chung WS, Lin CL, Kao CH. Diabetes increases the risk of deep-vein thrombosis and pulmonary embolism. A population-based cohort study. Thromb Haemost 2015;114:812-8.

23. Keller K, Rappold L, Gerhold-Ay A, et al. Sex-specific differences in pulmonary embolism. Thromb Res 2019;178:173-81.

24. Agarwal S, Clark D 3rd, Sud K, et al. Gender Disparities in Outcomes and Resource Utilization for Acute Pulmonary Embolism Hospitalizations in the United States. Am J Cardiol 2015;116:1270-6.

25. Blanco-Molina A, Enea I, Gadelha T. Sex differences in patients receiving anticoagulant therapy for venous thromboembolism. Medicine (Baltimore) 2014;93:309-17.

26. van Mens TE, van der Pol LM, van Es N, et al. Sexspecific performance of pre-imaging diagnostic algorithms for pulmonary embolism. J Thromb Haemost 2018;16:858-65.

27. Sanchez O, Trinquart L, Caille V, et al. Prognostic factors for pulmonary embolism: the prep study, a prospective multicenter cohort study. Am J Respir Crit Care Med 2010;181:168-73.

28. Lee YH, Cha SI, Shin KM, et al. Clinical relevance of syncope in patients with pulmonary embolism. Thromb Res 2018;164:85-9.

29. Goyard C, Côté B, Looten V, et al. Determinants and prognostic implication of diagnostic delay in patients with a first episode of pulmonary embolism. Thromb Res 2018;171:190-8.

30. Thames MD, Alpert JS, Dalen JE. Syncope in patients with pulmonary embolism. JAMA 1977;238:2509-11.

31. Simpson RJ Jr, Podolak R, Mangano CA Jr, et al. Vagal syncope during recurrent pulmonary embolism. JAMA 1983;249:390-3.

32. Sidelmann JJ, Kluft C, Krug AH, et al. Fibrin clot structure - pro-fibrinolytic effect of oral contraceptives in apparently healthy women. Thromb Haemost 2017;117:700-5.

Cite this article as: Yang YL, Yuan P, Wang CY, Pudasaini B, Li Y, Yu YZ, Zhao QH, Wang L, Gong SG, Jiang R, Wu WH, He J, Guo J, Luo CJ, Qiu HL, Chen C, Li JL, Liu JM. Variable predictors of acute pulmonary embolism recurrence with duration of follow-up. J Thorac Dis 2020;12(3):403-413. doi: 10.21037/jtd.2020.01.27 
A

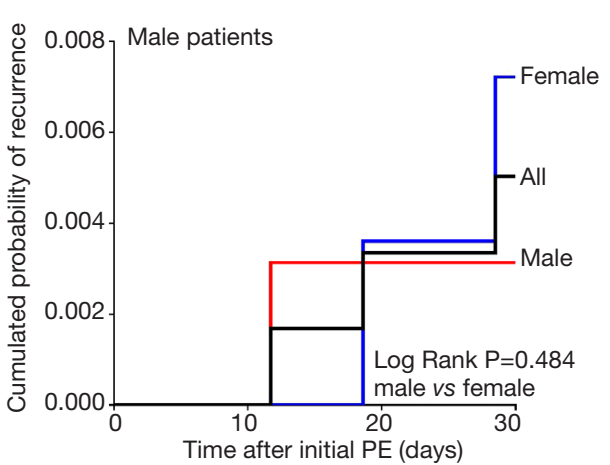

Number at risk

All 597

Male 319

Female 278

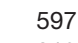

597
319
278

278

595

318
277

D

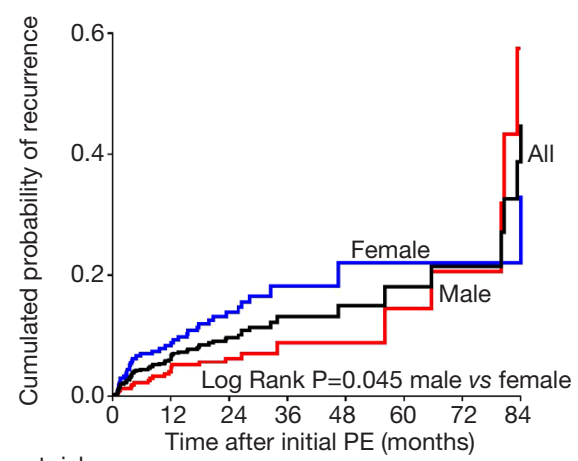

Number at ris

$\begin{array}{lllllllll}\text { All } & 597 & 438 & 276 & 62 & 40 & 26 & 17 & 9\end{array}$

Male $\begin{array}{llllllll}319 & 246 & 155 & 36 & 23 & 15 & 9 & 3\end{array}$

Female $\begin{array}{llllllll}278 & 192 & 121 & 26 & 19 & 11 & 8 & 6\end{array}$

\section{B}

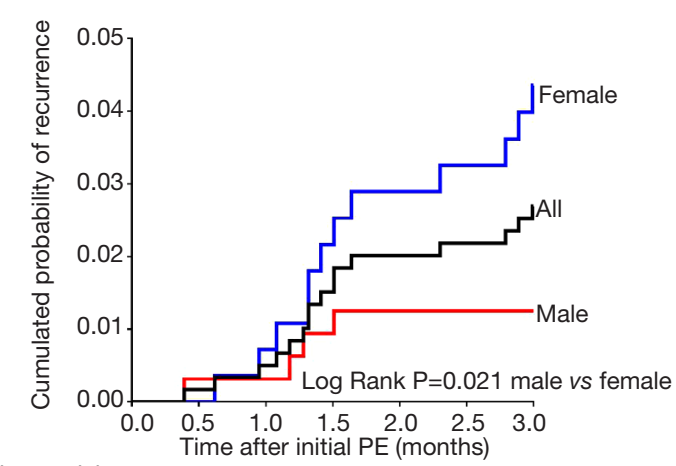

Number at risk

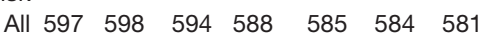

Male $\begin{array}{lllllll}319 & 318 & 318 & 315 & 315 & 315 & 315\end{array}$

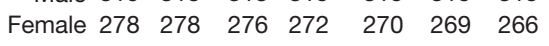

E

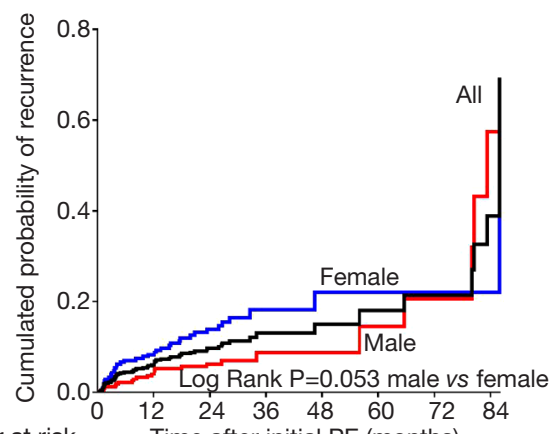

All $597438 \quad 276 \quad 62 \quad 40$ (months)

Male $\begin{array}{llllllll}319 & 246 & 155 & 36 & 23 & 15 & 9 & 3\end{array}$ Female $278 \begin{array}{lllllll}192 & 121 & 26 & 19 & 11 & 8 & 6\end{array}$

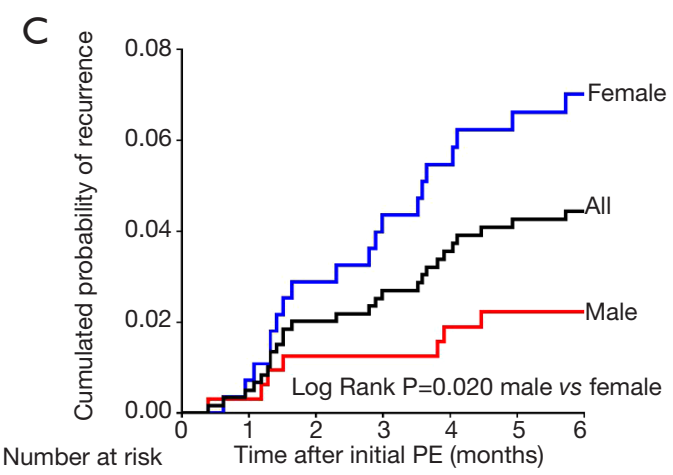

Number at risk Time after initial PE (months) All $597 \quad 594 \quad 589 \quad 581 \quad 576 \quad 572 \quad 571$ Male $\begin{array}{lllllll}319 & 318 & 315 & 315 & 313 & 312 & 312\end{array}$ Female $\begin{array}{lllllll}278 & 276 & 270 & 266 & 263 & 260 & 259\end{array}$

F

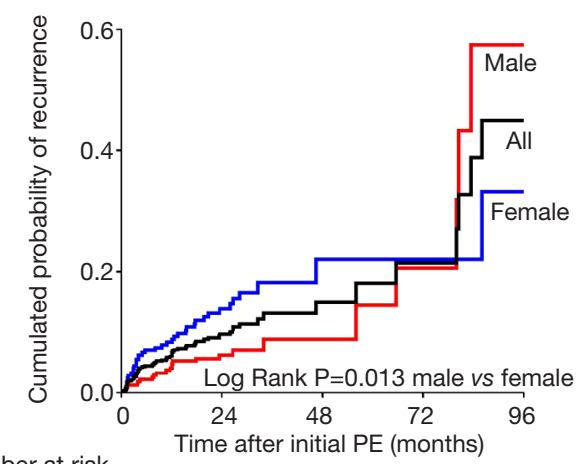

Number at risk

$\begin{array}{rllll}\text { All 597 } & 276 & 40 & 17 & 3 \\ \text { Male 319 } & 155 & 23 & 9 & 2 \\ \text { Female 278 } & 121 & 17 & 8 & 1\end{array}$

Figure S1 Sex disparities in cumulative recurrence-free survival curves and recurrence probability of patients with PE. (A) cumulative recurrence probability during 30-day followup; (B-F) cumulative recurrence probability at 3, 6, 84, 86 and 96-month follow-up periods. 
Table S1 Parameters predictive of recurrence probability in enter univariate and multivariate Cox proportional hazards analysis

\begin{tabular}{|c|c|c|c|c|c|c|}
\hline Time & Patients & Variables & \multicolumn{2}{|l|}{ Univariate } & \multicolumn{2}{|l|}{ Multivariate } \\
\hline \multirow[t]{3}{*}{ 30-day } & All & Diabetes & $11.274(1.022-124.343)$ & 0.048 & $18.796(1.707-207.315)$ & 0.017 \\
\hline & Male & - & - & - & - & - \\
\hline & Female & - & - & - & - & - \\
\hline \multirow{4}{*}{ 3-month } & & Acute infection & $0.274(0.082-0.906)$ & 0.034 & & \\
\hline & Male & Hyperlipemia & 6.798 (1.468-31.473) & 0.014 & $15.666(1.421-172.774)$ & 0.025 \\
\hline & Female & The history of DVT & $5.825(1.753-19.354)$ & 0.004 & $5.457(1.364-21.827)$ & 0.016 \\
\hline & & Acute infection & $0.191(0.040-0.920)$ & 0.039 & & \\
\hline \multirow{5}{*}{ 6-month } & & Hyperlipemia & 4.569 (1.038-20.115) & 0.045 & - & - \\
\hline & & The history of DVT & 3.185 (1.279-7.933) & 0.013 & - & - \\
\hline & Male & Hyperlipemia & $10.901(1.133-104.920)$ & 0.039 & - & - \\
\hline & Female & The history of DVT & $3.306(1.096-9.971)$ & 0.034 & - & - \\
\hline & & Acute infection & $0.305(0.106-0.879)$ & 0.028 & $0.305(0.106-0.879)$ & 0.028 \\
\hline
\end{tabular}

Values are median (interquartile range). $\dagger$, altered mental status was defined as disorientation, stupor or coma. $\mathrm{Cl}$, confidence interval; DVT, deep vein thrombosis; Log D-dimer, a logarithmic transformation of the D-dimer. 